\title{
Analysis of ERS-1/2 synthetic aperture radar wave mode imagettes
}

\author{
Vincent Kerbaol and Bertrand Chapron \\ Déparliement d'Océanographie Spatiale, IFREMER, Centre de Brest, Plouzané, France \\ Paris W. Vachon \\ Canada Centre for Remote Sensing, Ottawa, Ontario, Canada
}

\begin{abstract}
The European Space Agency (ESA) ERS-1 and ERS-2 C band VV polarization active microwave instrument (AMI) offers the unique ability to combine interlaced wind scatterometer and high-resolution synthetic aperture radar (SAR) wave mode imagettes. In this study, more than 2000 imagettes were considered. Each imagette has been statistically analyzed in comparison with normalized radar cross-section (NRCS) measurements from the scatterometer mode. During the ERS-1 AMI wave mode mission the incidence angle of the imagette center was modified from roughly $19.9^{\circ}$ to $23.5^{\circ}$. Using ERS-1 and ERS-2 NRCS, calibration has thus been completed for both configurations, which allows a better characterization of some signal data saturation effects. However, since a SAR relies on platform displacement to achieve fine resolution, surface motions reduce its nominal resolution. As the wind speed increases, scatterer motion occurring during the SAR integration time also increases, causing the characteristic large azimuth cutoff wavelength. Acknowledging the strong dependence between sea state conditions and azimuth smearing effects, our results are highlighted by the global comparison with wind estimates derived from the scatterometer. The results demonstrate the ability to define a SAR wind algorithm from a kinematic point of view. Finally, a higher-order statistical analysis shows evidence of deviation from standard Rayleigh statistics, leading to a balance between $\mathrm{K}$ law and lognormal distributions. This deviation is mainly due to the SAR's high-resolution properties.
\end{abstract}

\section{Introduction}

Since the launch of Seasat in 1978 it has been clear that synthetic aperture radar (SAR) instruments can image ocean surface wave patterns [Alpers et al., 1981; Vesecky and Stewart, 1982]. Unfortunately, it is now well known that the wave-like patterns visible in a SAR inlage of the ocean surface may be considerably different from the actual ocean wave field. As a result, extraction of meaningful two-dimensional wave spectral properties from a SAR scene is not straightforward. The capacity of SAR to provide usable ocean wave spectra is limited by the motion of the ocean surface. Although a polarorbiting SAR is not an ideal instrument to measure the directional spectrum of ocean surface waves, valuable information may be routinely extracted. In particular, with the ERS-1 and ERS-2 missions and the so-called SAR wave mode products [European Space Agency, 1992a], wave modelers can, for the first time, obtain continuous global information [Brüning et al., 1994].

Copyright 1998 by the American Geophysical Union.

Paper number 97JC01579.

0148-0227/98/97JC-01579\$09.00
Wave mode data continuity will be available through ENVISAT.

As an attempt to extract meaningful information from ocean SAR scenes, we have focused on the unique ability of the ERS active microwave instrument (AMI) to combine interlaced scatterometer and SAR wave mode measurements. The wave mode is discussed in section 2. Our objectives are met by carrying out a systematic comparison between normalized radar crosssection (NRCS) data and wind vector estimates from the scatterometer, with various statistical and observed spectral parameters of collocated SAR imagettes.

A primary measurable of a calibrated SAR is backscattered power. This measurement is diffraction dominated and depends upon short sea surface wind waves. Thus an important capability of a SAR instrument should be its ability to quantitatively relate image intensity to local surface wind speed. Empirically derived wind retrieval algorithms are now routinely used to infer wind estimates from scatterometer and altimeter, but much less attention has been devoted to SAR instruments [Vachon and Dobson, 1996]. In section 3 we present a calibration procedure to use SAR data much like a higher-resolution scatterometer. In the absence of 
specific information for SAR imagette radiometric analysis our method is to derive a relative calibration from direct global comparisons between collocated SAR and scatterometer central antenna measurements. The relative calibration follows a strong saturation law. Thus the relationship between scatterometer central antenna NRCS and mean values of imagette intensity must take into account saturation of the analog-to-digital converter $(A D C)$. In particular, this study shows evidence of the change of imagette incidence angle during the ERS-1 mission. The validation of this empirical calibration process is presented in section 4 using a semiempirical wind retrieval scattering model, which has been developed at the Institut Français de Recherche pour l'Exploitation de la Mer (IFREMER) and referred as CMOD-IFREMER, [Quilfen, 1993].

Improved information has also been obtained through global and systematic analysis of the SAR along-track resolution, i.e., the azimuthal cutoff. According to theory, this latter parameter is proportional to the rootmean-square (rms) line of sight of the wave orbital velocity field. Since the ERS-1 and ERS-2 wave mode operate at near-nadir incidence angles $\left(19.9^{\circ}\right.$ and $\left.23.5^{\circ}\right)$, the radial component is supported by the surface vertical velocity which, by definition, will be dominated by shorter gravity waves than the overall energy integral [Jackson and Peng, 1985]. The portion of the spectrum supporting these waves is usually assumed to be wind dependent, and we propose that the azimuthal response is wind speed dependent. Early results based on global comparisons confirmed this assumption [Chapron et al., 1995]. In section 5, azimuth cutoff is discussed in terms of incidence angle, wind directionality, and sea state development.

From a statistical point of view, direct analysis of high-resolution radar scenes over the ocean exhibits departures from a standard Gaussian-Rayleigh model.
This deviation is demonstrated in section 6 through analysis of higher-order moments and is represented in a Pearson diagram, which is a convenient representation of distribution families. The source of this deviation is discussed in light of SAR high-resolution properties by studying SAR power image variance behavior. Indeed, the concept of large-scale inhomogeneities (long wave field) modulating a dense array of small-scale scatters can lead to non-Gaussian statistics.

\section{ERS Wave Mode Data}

The CERSAT (Centre for Archiving and Processing of ERS data), located at IFREMER in Brest, is tasked with archiving SAR wave mode imagettes and processing scatterometer data for the ERS-1 and ERS-2 satellites. It provides the unique opportunity to combine the analysis of both products.

The European Space Agency (ESA) ERS-1 and ERS$2 \mathrm{C}$ band VV polarization AMI offers the ability to obtain interlaced scatterometer data and SAR wave mode imagettes (Figure 1). The $10 \times 5 \mathrm{~km}$ imagettes are range compressed on board the satellite and are interlaced with scatterometer data every $200 \mathrm{~km}$ along the satellite track. These imagettes represent magnitude look detection and incoherent averaging of three independent looks. Each imagette consists of 600 pixels in range (across track) and 320 pixels in azimuth (along track), with pixel spacing of $20 \mathrm{~m}$ in range and $16 \mathrm{~m}$ in azimuth.

In this work, more than 2000 imagettes have been considered, representing a wide variety of conditions. Various statistics have been measured, including NRCS, azimuth cutoff, and higher-order statistics. Our results are highlighted by the global comparison with calibrated NRCS and wind estimates derived from the collocated scatterometer measurements.

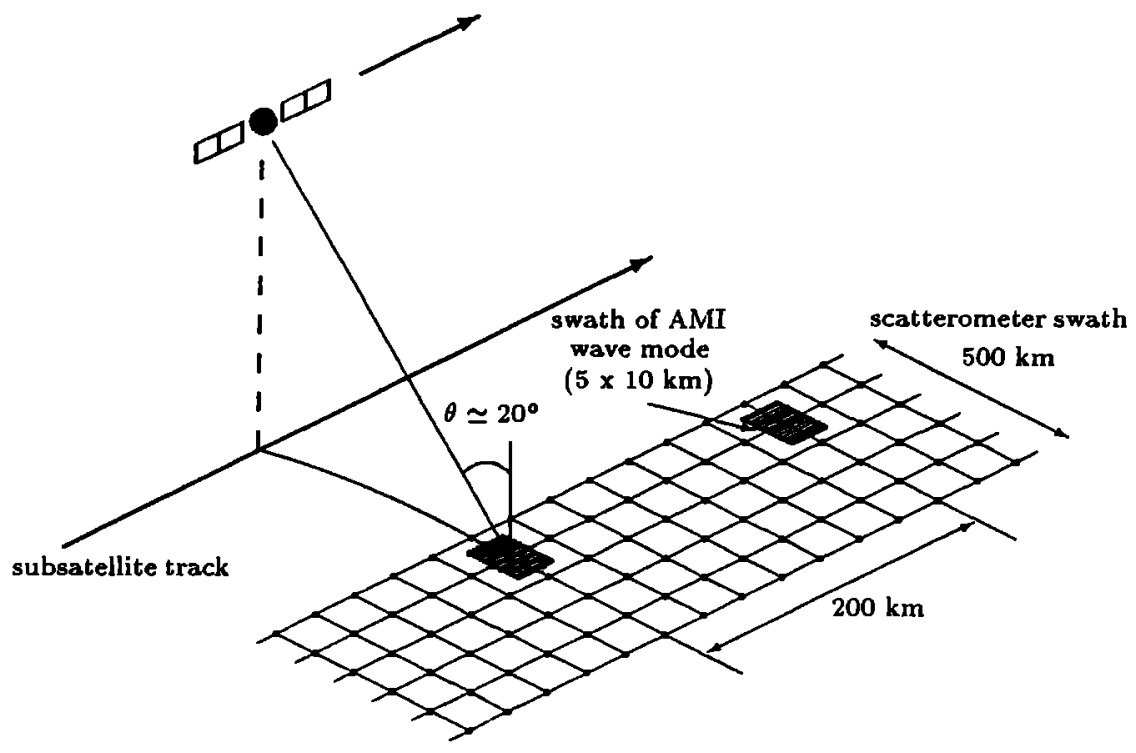

Figure 1. ERS synthetic aperture radar (SAR) wave mode and scatterometer geometry. 


\section{Calibration}

Ocean ripples are directly related to local sea surface wind velocity and can be detected by radar techniques. In particular, SAR scattering measurements are diffraction dominated and depend upon short gravity-capillary wind waves. Thus an important capability of a SAR instrument is its ability to quantitatively relate image intensity to local surface wind speed. Empirically derived wind retrieval algorithms are now routinely used to infer wind estimates from scatterometer and altimeter data. Much less attention has been devoted to SAR instruments [Scoon et al., 1996; Vachon and Dobson, 1996]. If the SAR is used like a high-resolution scatterometer to derive wind speed estimates from an empirically derived satellite wind model function such as CMOD, we need to relate pixel values in the imagettes to NRCS values.

SAR imaging of the ocean surfaces also relies on Doppler information from the relative motion between the SAR and the scene to achieve fine resolution in the along-track direction. For example, the SAR images waves by their own orbital velocities. Using the concept of facets, the effect of long waves (longer than the nominal SAR resolution cell) can be treated deterministically [Alpers and Ruffenach, 1979; Swift and Wilson, 1979] whereas short waves (shorter than the SAR resolution cell) may be treated stochastically [Tucker, 1985]. The motions due to short waves induce an azimuth smearing that acts as a low-pass filter (often modeled as Gaussian-shaped) on the azimuthal components of the image spectrum. In particular, it has been stated that this imaging process turns into a superposition of facets [Hasselmann and Hasselmann, 1991]. The study of the number of superposed facets has not been considered in detail [Kanevsky, 1993; Chapron et al., 1994a]. However, this phenomenon should not influence the mean image intensity level because of the conservation of the mean between the real aperture radar (RAR) and the SAR image [Hasselmann and Hasselmann, 1991].

From the radar equation the SAR image intensity may be written

$$
I_{\mathrm{SAR}}=\frac{K \sigma^{\circ} g^{2}(\theta)}{R^{3} \sin \theta} \operatorname{Loss}(\mathrm{ADC}, \cdots)
$$

where $K$ represents a calibration constant, $\sigma^{\circ}$ represents the radar cross-section, $\theta$ represents the incidence angle, $R$ represents the slant range, $g$ represents the two-way elevation antenna pattern, and Loss represents a function accounting for any power loss processes (such as ADC saturation). As delivered, imagettes are not corrected for range spreading and antenna pattern. Yet these corrections may be considered as corrective constants due to the small incidence angle range over each imagette $\left(0.8^{\circ}\right)$. The radar equation is simplified as

$$
I_{\mathrm{dB}}=\sigma_{\mathrm{dB}}^{\circ}+K_{\mathrm{dB}}^{\prime}+\operatorname{Loss}_{\mathrm{dB}}
$$

with $K_{\mathrm{dB}}^{\prime}$, a modified calibration constant, defined as

$$
K_{\mathrm{dB}}^{\prime}=K_{\mathrm{dB}}+10 \log _{10}\left[g^{2}(\theta)\right]-10 \log _{10}\left(R^{3} \sin \theta\right)
$$

\subsection{ADC Saturation}

The quantization of $I$ (real) and $Q$ (imaginary) signal data channels within the ADC on a limited number of bits is known to reduce the output power. For ERS SAR Precision Images (PRI) [European Space Agency, 1992b], both channels are quantized with 5 bits [Laur et al., 1993]. For SAR imagettes, quantization is applied on 4 bits [Kerbaol and Chapron, 1996]. Radar cross-section measurements are then strongly affected by ADC saturation when the backscattered power takes large values ( $>-6 \mathrm{~dB}$ ), which corresponds to wind speeds greater than $6 \mathrm{~m} / \mathrm{s}$ for range winds and $10 \mathrm{~m} / \mathrm{s}$ for azimuthal winds. The power loss correction is given as a function of the $I$ or $Q$ channel standard deviation (Figure 2). The curve was obtained by simulation, assuming the $I$ and $Q$ channels to be normally distributed.

Fortunately, ADC power loss correction can be applied on ERS-1/2 imagettes even though raw data are not at our disposal. Indeed, the I/Q channel standard deviation is provided in the header of each imagette, and the resulting power loss can be corrected on the entire imagette since, in most cases, the homogeneity of the imagette is assured by its small size (5 $\times 10 \mathrm{~km})$. Note that the ADC saturation effect is not expected to significantly affect the mean SAR intensity of ERS-2 imagettes because of a reduction in onboard gains in July 1995 [Laur et al., 1996].

\subsection{Change of the ERS-1 SAR Incidence Angle From $19.9^{\circ}$ to $23.5^{\circ}$}

The calibration of ERS-1/2 imagettes would be complete if the calibration constant $K$ is known. Though this constant is well known for PRI images, it is not available for wave mode products, which makes the cal-

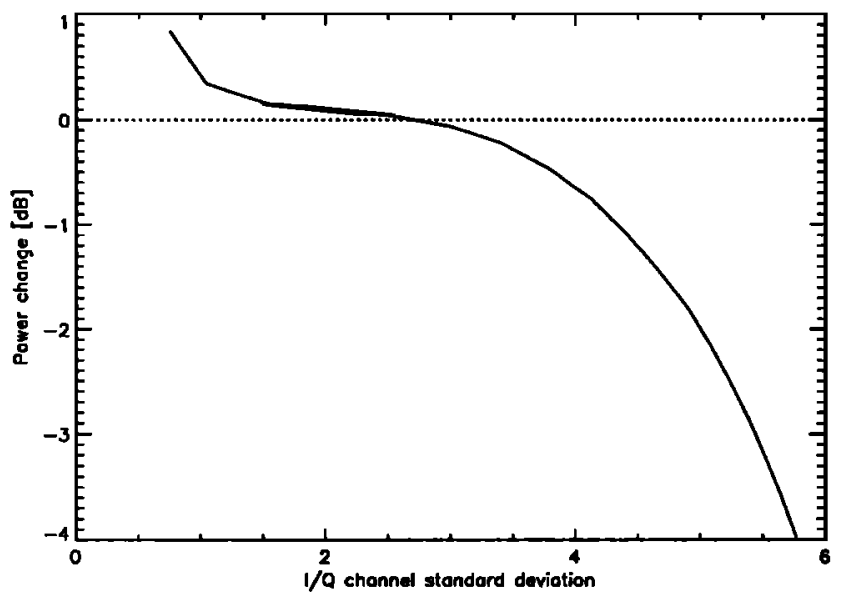

Figure 2. Power loss at analog-to-digital converter (ADC) output versus $I$ or $Q$ channel standard deviation for a 4 bit ADC. 
ibration issue more difficult. An approach to wave mode calibration is to compare the mean intensity of ERS-1/2 SAR imagettes to the collocated scatterometer NRCS. Then, after subtracting $K_{\mathrm{dB}}^{\prime}$ from the mean imagette intensity after correction for ADC power loss, one should obtain a 1-to-1 ratio with scatterometer $\sigma^{\circ}$.

This method enabled us to refine the exact incidence angle used to acquire the ERS-1/2 SAR wave mode products. For ERS-2 imagettes the incidence angle is set to $23.5^{\circ}$. This was also meant to be the incidence angle for ERS-1. However, it was originally set to $19.9^{\circ}$. In June 1995 the ERS-1 imagette incidence angle was reset to $23.5^{\circ}$. Two data sets were chosen to illustrate this change of configuration. The first one represents 1092 imagettes acquired in November 1992 over the North Pacific Ocean, while the second one represents 693 imagettes acquired in June 1995 over the Indian Ocean (Figure 3).

Figure 4a shows the comparison result with collocated scatterometer NRCS at $19.9^{\circ}$ (November 1992), while Figure $4 \mathrm{~b}$ shows the result at $23.5^{\circ}$ (June 1995). As expected, the change in incidence angle decreases the mean $\sigma^{\circ}$ value and results in a reduction of the ADC saturation effect.

After ADC saturation correction the expected 1-to-1 ratio is obtained for the $23.5^{\circ}$ incidence angle data set, whereas it is only reached for the $19.9^{\circ}$ data set for the lowest measured cross sections. Beyond a certain level, saturation is so large that it becomes difficult to correct for power loss. Note the spreading of data around the 1-to-1 ratio line for the smallest scatterometer NRCS values. This can be associated with the much larger scatterometer resolution cell $(25 \times 25 \mathrm{~km})$ compared to the SAR $(30 \times 30 \mathrm{~m})$. Indeed, the variability at low wind speed is integrated and smoothed over the large scatterometer resolution cell while SAR imagettes only provide local measurements.

\subsection{ERS-2 Onboard Gain Adjustment}

For ERS-2 SAR imagettes a set of 342 imagettes, acquired over the Indian Ocean in June 1996, was selected (Figure 3). Figure 5 shows that the imagettes are almost not affected by ADC saturation. This is due to a reduction in ERS-2 onboard gains on July 13, 1995. ADC saturation correction is not essential, except for $\sigma^{\circ}$ values larger than $-3 \mathrm{~dB}$. This level is close to the -2 dB level given by Laur et al. [1996, p. 11] for ERS-2 PRI images. All the $K^{\prime}$ values for ERS-1 and ERS-2 are given in the appendix $A$.

\section{Validation}

Recent studies [Scoon et al., 1996; Vachon and Dobson, 1996] have shown the SAR ability to work as a high-resolution scatterometer to retrieve wind speed using a scatterometer wind model (CMOD4 [Stoeffelen and Anderson, 1993] or CMOD IFREMER [Quilfen, 1993]). The method is to compare SAR range profiles to a wind retrieval model assuming a known, steady wind vector.

Here we propose to validate our empirical calibration method. For each data set we selected cases whose wind directions, as given by ERS scatterometer, are either in the range or the azimuth direction. The corresponding mean SAR intensity is then compared with CMOD IFREMER. Figure 6 shows the results for ERS-

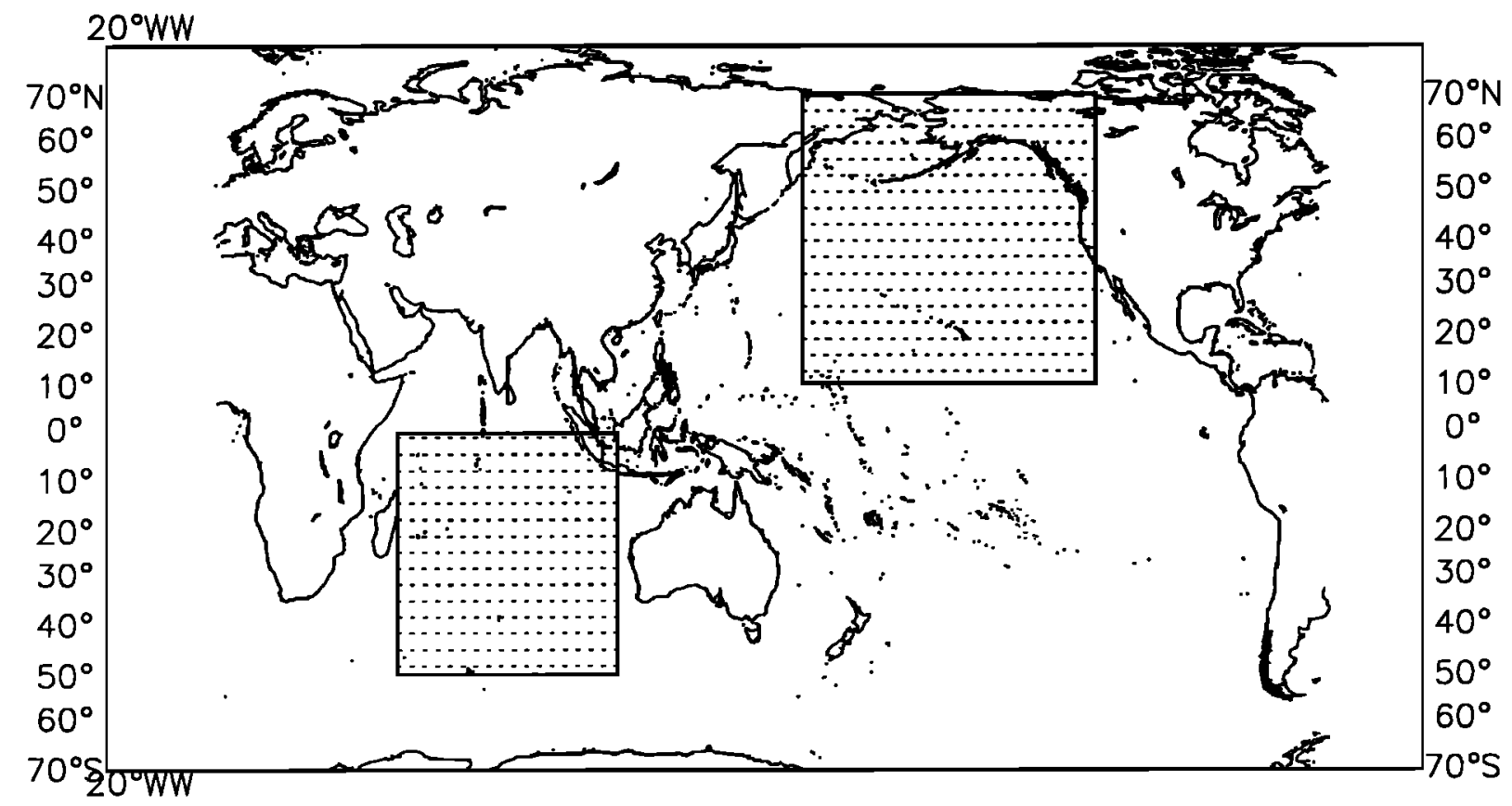

Figure 3. Areas selected for ERS-1 imagettes: North Pacific Ocean (November 1992) and Indian ocean (June 1995). Area selected for ERS-2 imagettes: Indian Ocean (June 1996). 


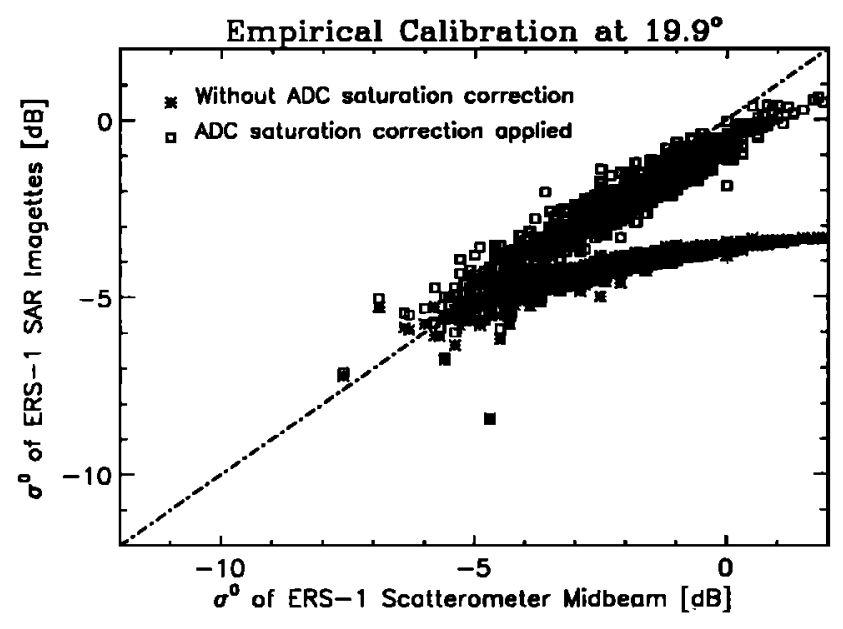

(a)

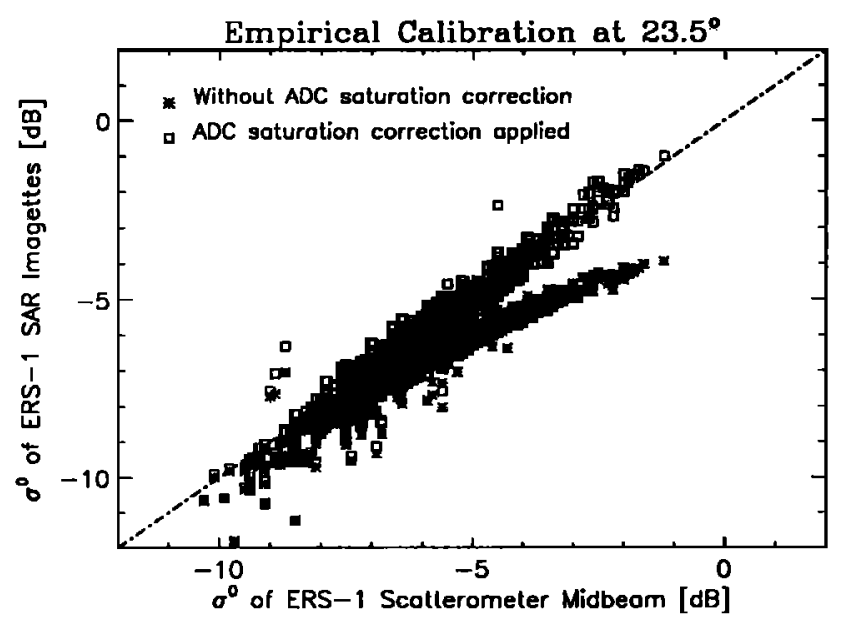

(b)

Figure 4. ERS-1 mean SAR intensity versus collocated scatterometer $\sigma^{\circ}$ before ADC correction (asterisks) and after correction (squares). The line represents a 1-to-1 ratio. The calibration constant $K^{\prime}$ (Table A1) has been subtracted from the SAR intensity. (a) North Pacific Ocean data set $\left(\theta=19.9^{\circ}\right.$, November 1992). (b) Indian Ocean data set $\left(\theta=23.5^{\circ}\right.$, June 1995).

1 SAR in both incidence angle configurations $\left(19.9^{\circ}\right.$ (Figure 6a) and $23.5^{\circ}$ (Figure 6b)). One can see that ERS-1 SAR imagettes have been successfully calibrated for the incidence angle of $23.5^{\circ}$. Indeed, both wind directions (along range and azimuth) lie on the respective CMOD IFREMER curve. However, this is not the case for the data set over the North Pacific Ocean, particularly when the wind speed increases. This is due to the weakness of the ADC saturation correction at $19.9^{\circ}$. Incidentally, it appears that the wind retrieval model used for this study tends to underestimate high wind speeds, particularly in the range direction (up/down winds). Winds are expected to be as high as $18 \mathrm{~m} / \mathrm{s}$, rather than the maximum $15 \mathrm{~m} / \mathrm{s}$ of Figure $6 \mathrm{~b}$. The results for ERS-2 imagettes are presented in Figure 7. The calibration scheme also gives good results for ERS-2 SAR wave mode imagettes, although only a few examples of azimuthal wind were at our disposal. Indeed, winds generally blow from west to east in the roaring forties (e.g., mainly along the range direction).

According to these comparisons, the SAR instrument may prove its ability to work as a high-resolution scatterometer. Given a first-guess wind field direction (such as provided by a meteorological model or synoptic chart), the wind speed can be obtained from SAR images using the CMOD retrieval algorithm. Inversely, from an independent wind speed measurement a properly calibrated SAR signal might be able to provide the wind direction [e.g., Korsbakken, 1996].

\section{SAR Azimuth Cutoff}

Doppler misregistrations in azimuth are induced by gravity wave orbital motion, leading to a distortion of the imaged spectrum and a strong cutoff in the azimuthal direction. This effect is proportional to the range-to-platform velocity ratio $R / V$ [Beal et al., 1983]. ERS-1, ERS-2, RADARSAT, and planned future SAR missions such as ENVISAT are polar-orbiting platforms and have an $R / V$ of about $120 \mathrm{~s}$ or larger. Then, depending on sea state, the shortest detectable wavelength in the azimuth direction will not be less than $200 \mathrm{~m}$. We calculated SAR image spectra for all ERS imagettes of our data set and plotted the spectral peak wavelength against its direction relative to the range axis (Figure 8). This plot shows that all waves shorter than $100 \mathrm{~m}$ can only be observed in the range direction. Note that only a few peaks arise right on the range axis. This is due to the so-called peak-splitting effect [Brüning et al., 1990]. The effect of SAR mapping is to low-pass fil-

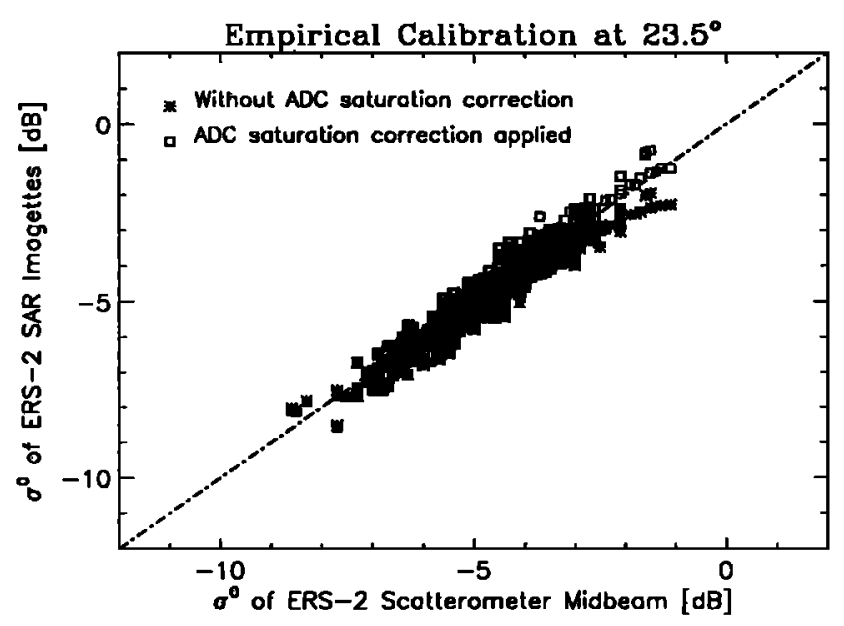

Figure 5. ERS-2 mean SAR intensity versus collocated scatterometer $\sigma^{\circ}$ before ADC correction (asterisks) and after correction (squares). The line represents a 1-to-1 ratio. The calibration constant $K^{\prime}$ (Table A1) has been subtracted from the SAR intensity. Indian Ocean data set $\left(\theta=23.5^{\circ}\right.$, June 1996). 


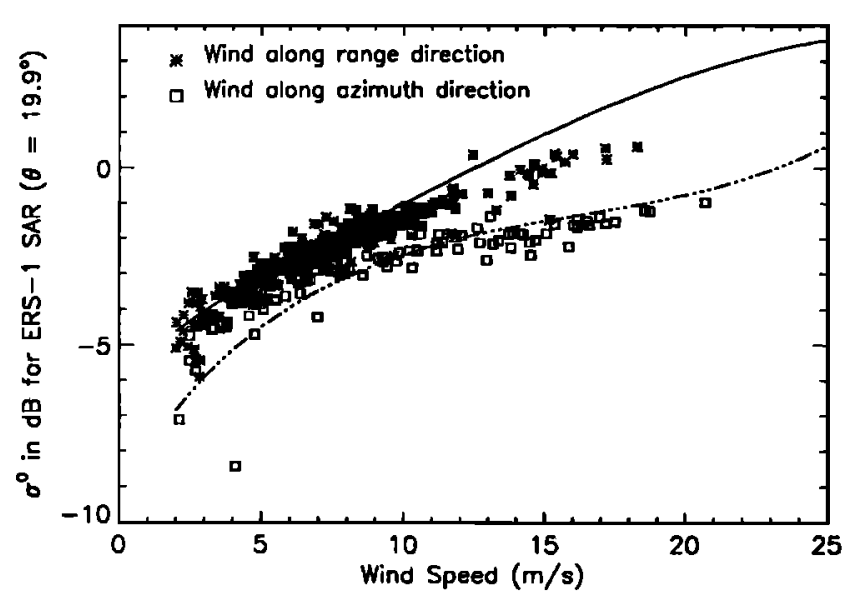

(a)

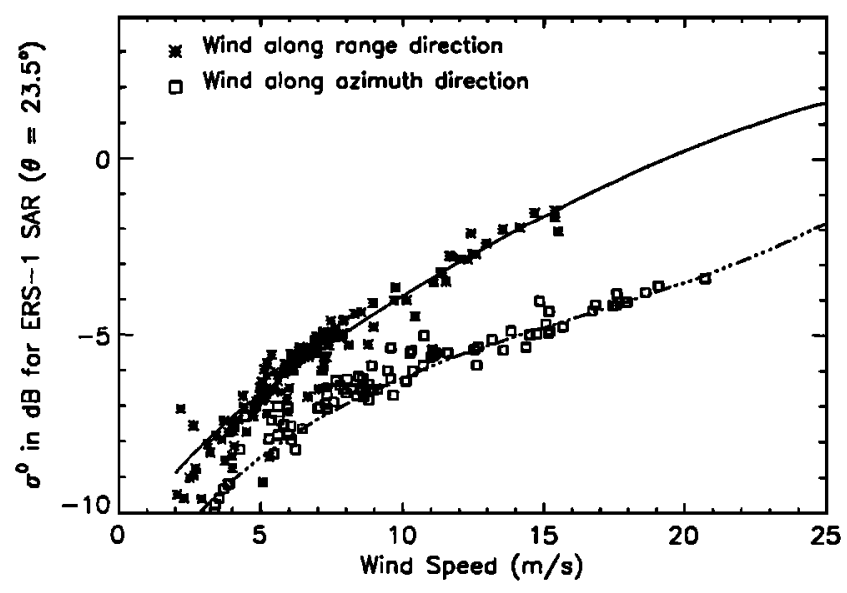

(b)

Figure 6. ERS-1 SAR $\sigma^{\circ}$ versus wind speed given by ERS-1 scatterometer for wind blowing in the range direction (asterisks) and in the azimuth direction (squares). The solid curve represents the empirical $\mathrm{C}$ band scattering model (CMOD-IFREMER) $\sigma^{\circ}$ for a range wind direction and the dot-dashed curve for an azimuth wind direction. (a) North Pacific Ocean data set $\left(\theta=19.9^{\circ}\right.$, November 1992). (b) Indian Ocean data set $\left(\theta=23.5^{\circ}\right.$, June 1995).

ter the real aperture radar (RAR) spectral information in the azimuthal direction such that the SAR spectral domain is limited to around the range axis.

The issue of the SAR azimuth cutoff interpretation has been widely considered in the literature, and several models have been proposed as a function of wave and/or wind conditions. A first interpretation was initially proposed in terms of the significant wave height $H_{S}$ [Beal et al., 1983], but the azimuth cutoff has been shown to also be wind speed dependent. Then the contribution of azimuthal displacements caused by the long waves orbital motions was outlined [Jackson and Peng, 1985; Tucker, 1985; Hasselmann et al., 1985] and appeared to be more realistic when compared with the cutoff behavior [Vachon et al., 1994]. Referring to studies on processes moving in time [Steinberg et al., 1955; Brillinger, 1993] and taking advantage of wind vector estimates inferred from collocated ERS scatterometer data, we will show evidence of the SAR azimuth cutoff dependence with the total variance of vertical motions on the sea surface.

The power spectrum of a signal whose source is subject to random fluctuations has already been studied and has been shown to be filtered by the characteristic function of the displacement field [Brillinger, 1993]. Hence, extending this relationship to forward SAR mapping while under the simplifying assumption that the displacement field and the linear SAR modulation field (including RAR and velocity bunching modulation) are independent, the relationship between $P_{\text {in }}(k)$, the power spectrum of the linear SAR transform of ocean spectrum, and $P_{S}(k)$, the complete SAR power spectrum, is

$$
P_{S}(k)=e^{-k_{a z}^{2} \rho_{\xi \varepsilon}(0)} P_{\text {lin }}(k)
$$

where $\rho_{\xi \xi}(0)$ represents the total variance of azimuthal displacements within the SAR integration time. This exponential weighting function is identical to the standard azimuthal cutoff factor [Hasselmann and Hasselmann, 1991; Krogstad, 1992]. This relationship is often referred to as the so-called quasi-linear approximation, and this transform behaves like an azimuthal low-pass filter in the spectral domain. Noting that the azimuthal displacement $\xi$ induced by a moving scatterer with radial velocity $v$ is

$$
\xi=\frac{R}{V} v
$$

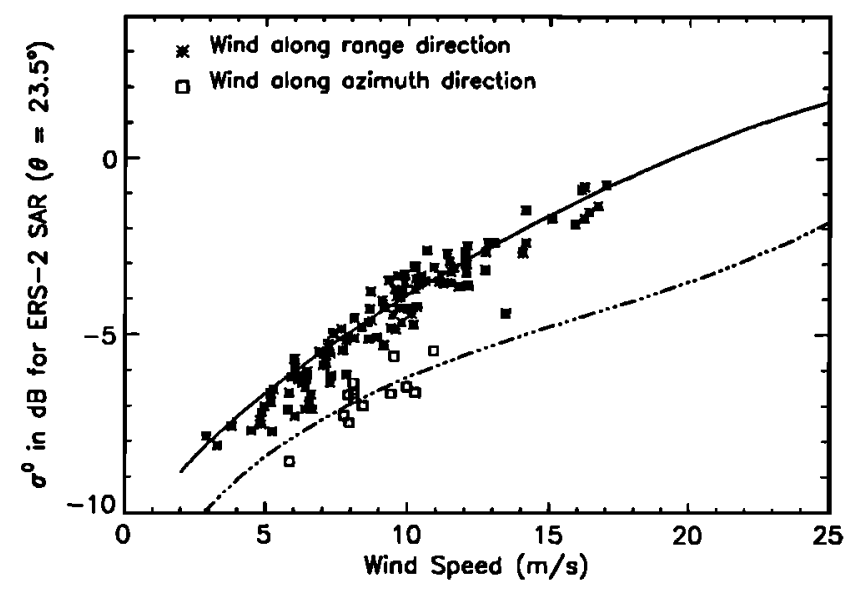

Figure 7. ERS-2 SAR $\sigma^{\circ}$ versus wind speed given by ERS-2 scatterometer for wind blowing in range direction (asterisks) and in azimuth direction (squares). The solid curve represents CMOD IFREMER $\sigma^{\circ}$ for a range wind direction and the dot-dashed curve for an azimuth wind direction. Indian Ocean data set $\left(\theta=23.5^{\circ}\right.$, June 1996). 


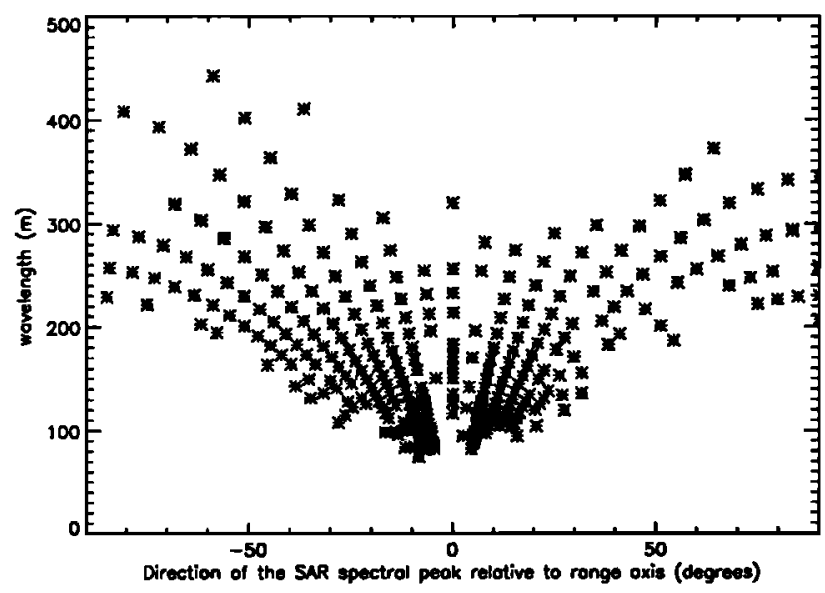

Figure 8. Wavelengths of observed SAR spectral peak versus the angle between $S A R$ peak and range axis.

and that at an incidence angle of about $20^{\circ}, v$ is well approximated by the vertical component of the orbital velocity (i.e., the directivity of the wave field can be neglected), $\rho_{\xi \xi}(0)$ becomes

$$
\rho_{\xi \xi}(0)=\left(\frac{R}{V}\right)^{2} \int_{0}^{\infty} \omega^{2} S(k) d k
$$

where $\omega$ is the wave frequency. Here $S(k)$ is integrated over the full wavenumber range in order to include the Doppler spread of the backscattered signal caused by the motion of the facets within a resolution cell. This additional azimuth smear is often expressed in terms of a coherence time.

One should note that $\rho_{\xi \xi}(0)$ does not take into account the radial acceleration term. Since the integration time of the ERS SARs is small (about $0.5 \mathrm{~s}$ ), the acceleration contribution can be neglected [Bao et al., 1994]. However, in some cases the smearing due to acceleration may become important. This is expected for high sea state cases when specular events, such as those from breaking waves, may lead to large azimuthal streaks and an enhancement of the azimuth cutoff [Alpers and Brüning, 1986; Ouchi and Cordey, 1991].

\subsection{Azimuth Cutoff Estimation}

An estimation method of the SAR azimuth cutoff has previously been presented [Vachon et al., 1994]. This procedure used an ensemble of model functions and various cutoff wavelengths to find the best fit between generated and observed SAR azimuth spectra. In order to gain in computing time we directly use the azimuthal autocorrelation function (ACF) by fitting a Gaussian function defined as

$$
C(x) \sim e^{-\pi^{2} \frac{x^{2}}{\lambda^{2}}}
$$

Generally speaking, we have found that this method gives good results. However, one needs to be cautious with the interpretation of the estimated cutoff. In the case of an imagette without any visual contrast, the ACF will be very close to the narrow point spread function associated with the speckle noise. When the sea surface motion increases, the ACF can then be considered as the sum of two spread functions, corresponding to the azimuthal resolution and the azimuth cutoff, respectively (Figure 9a).

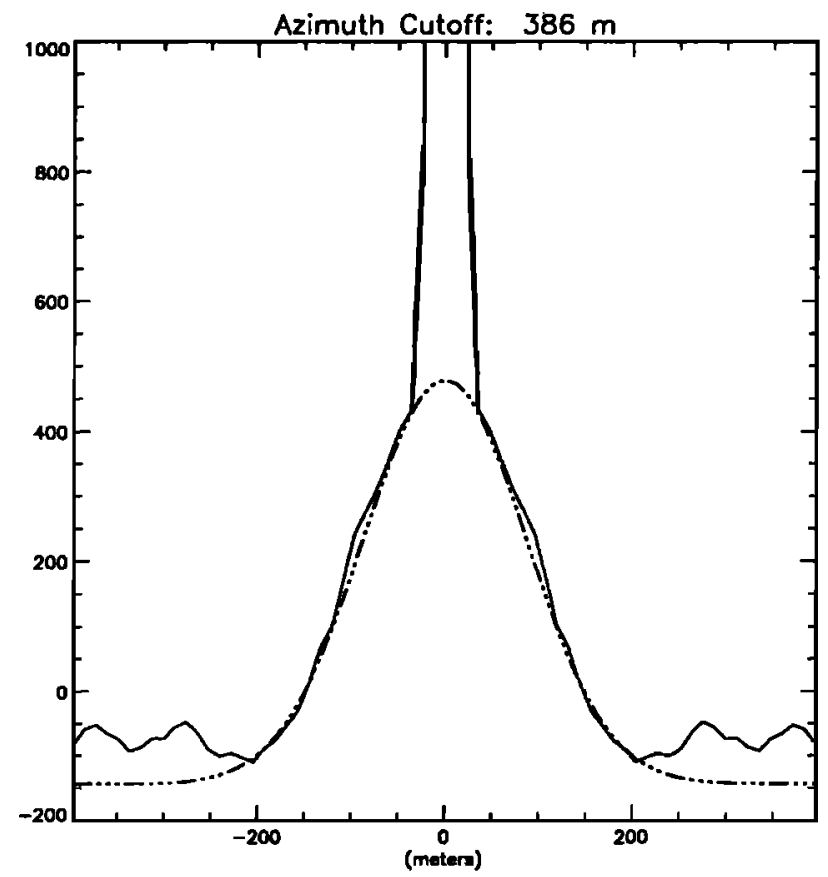

(a)

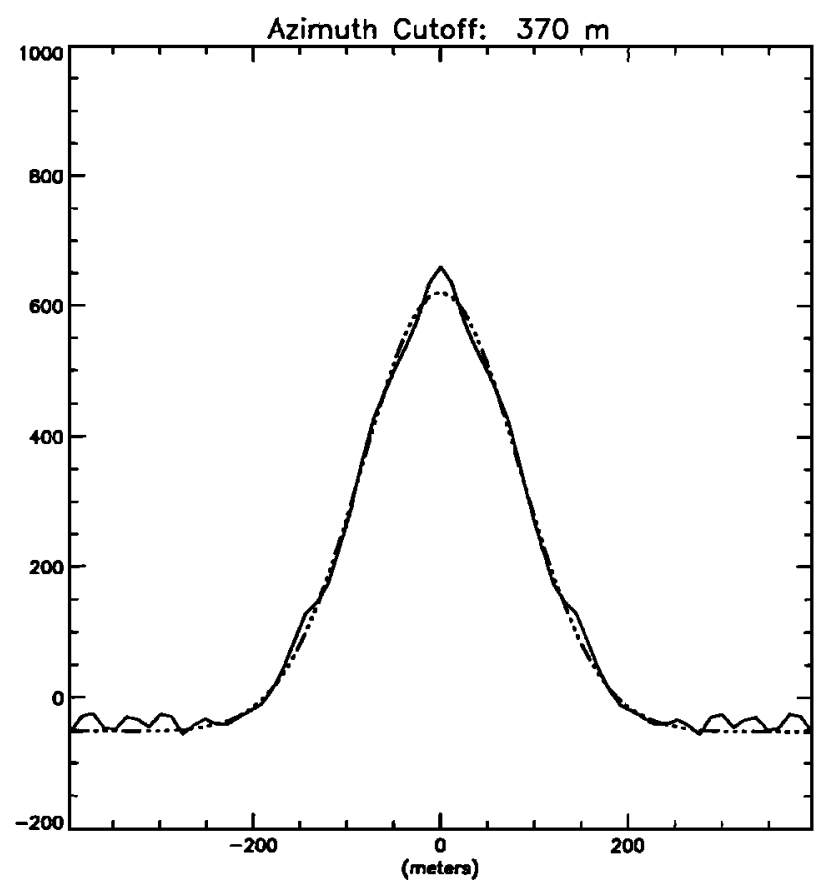

(b)

Figure 9. (a) Estimation of the azimuth cutoff through the azimuthal correlation function. (b) The narrow central peak of the non coherent scattering has been removed by using intercorrelation between two independent looks. 
In the near future the intercorrelation method between distinct looks may also be used. Indeed, when carrying the cross-spectrum of different looks, the incoherent scattering contribution will be suppressed [Engen and Johnsen, 1995]. The speckle noise contributes a large bandwidth noise floor (pedestal) in the SAR spectrum. The usual way to reduce the noise contribution is to use the multilook technique, but the azimuthal resolution is then degraded. The cross spectrum technique will lead to a much better signal-to-noise ratio by eliminating the uncorrelated noise. As a consequence, this technique is helpful when carrying out the intercorrelation between looks to estimate the falloff parameter. As noted above, when one observes the correlation function of a multilook SAR image, there is still an apparent contribution of the noise in the narrow peak of the speckle autocorrelation function. The intercorrelation function between different looks will not show this peak (Figure 9b). The estimation of the cutoff parameter will then become more consistent, especially under low wind conditions. Furthermore, the acceleration term in the azimuth smearing due to short lifetime specular events should not appear in the intercorrelation method.

\subsection{SAR Wind Algorithm}

Acknowledging the strong dependence between sea state conditions and azimuth-smearing effects, Jackson and Peng [1985] first suggested derivation of a wind algorithm from azimuth cutoff analysis. Global comparisons can now be done by using wind estimates derived from the ERS-1 or ERS-2 scatterometers. For each imagette the wind speed is obtained from the surrounding area (within $25 \mathrm{~km}$ ). Linear regression gives a convincing idea of a possible empirical relationship to define a wind algorithm from ACF analysis of SAR image spectra (Figure 10). Practical application of a linear relationship has already been demonstrated [ $\mathrm{Ko}-$ rsbakken, 1996]. If the SAR azimuth cutoff is expressed as [Lyzenga, 1986]

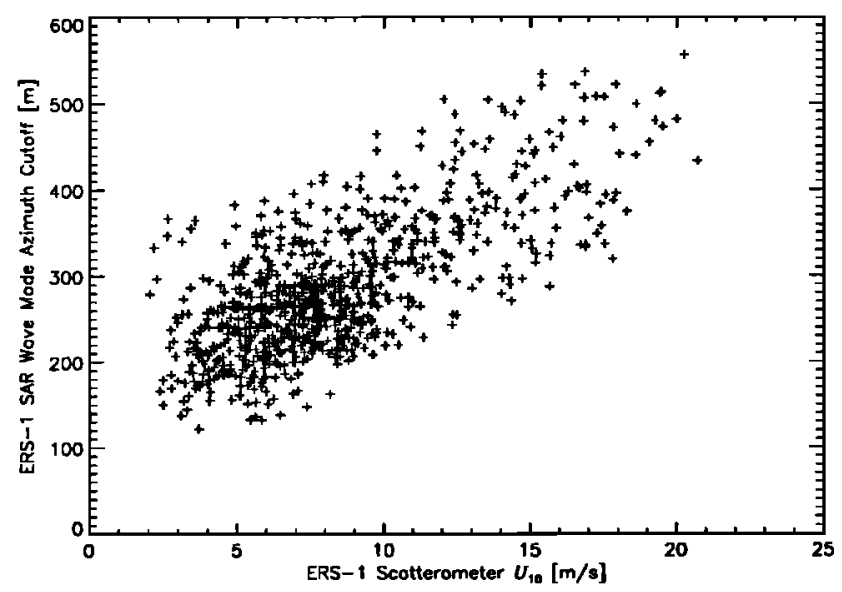

Figure 10. ERS-1 SAR azimuth cutoff versus collocated $U_{10}$ inferred from ERS-1 scatterometer.

$$
\lambda=\pi \sqrt{\rho_{\xi \xi}(0)}=\pi \frac{R}{V} \sqrt{\int_{0}^{\infty} \omega^{2} S(k) d k}
$$

a numerical application using a JONSWAP-like sea spectral model [Elfouhaily et al., 1996] leads to the linear relationship $\lambda \simeq 25 U_{10}$, where $U_{10}$ is the neutral stability wind speed at $10 \mathrm{~m}$ above the ocean surface. This evaluation is consistent with the result of our linear regression

$$
\lambda=23.4 U_{10}+\Lambda
$$

where $\Lambda$ represents a residual cutoff involving the nominal SAR azimuthal resolution. Note that this relationship is consistent with the analysis of Vachon et al. [1994] from the Grand Banks ERS-1 SAR validation experiment but with a different notation.

5.2.1. Wave age dependency. Although the kinematic parameter $\lambda$ is a robust wind speed estimator, it is necessary to consider that the degree of sea state development may also play a significant role. For example, a cutoff analysis over fetch-limited seas results in an underestimation of the wind speed [Kerbaol et al., 1996]. In this case we rewrite (8) as

$$
\lambda(\Omega)=\pi \sqrt{\rho_{\xi \xi}(0)}=\pi \frac{R}{V} \sqrt{\int_{0}^{\infty} \omega^{2} S(k, \Omega) d k}
$$

where $\Omega$ represents the inverse wave age dependency of the wave spectral model defined as [Elfouhaily et al., 1996]

$$
\Omega=0.84 \tanh \left[\left(\frac{k_{0} x}{2.210^{4}}\right)^{04}\right]^{-0.75}
$$

with $x$, the dimensional fetch in meters, and $k_{0}$ is $g / U_{10}^{2}$.

As an example, and following the wind and fetchdependent sea spectrum [Elfouhaily et al., 1996], Figure 11 shows the behavior of the theoretical $\lambda$ for wind speeds ranging from 2 to $25 \mathrm{~m} / \mathrm{s}$ given different fetch values. For our purpose we also consider that the degree of development is variable over different ranges of wind speed. If it is reasonable to take a fully developed model for light to moderate wind speed conditions, this will not in general be the case for high wind speeds. Following studies using altimeter data [Tournadre and Blanquet, 1994], we choose an average fetch of $200 \mathrm{~km}$ for the whole range of wind speed over the globe to illustrate this effect, which can be thought of as a saturation phenomenon. This corresponds to an inverse wave age parameter $\Omega$ of about 0.84 for low wind speed to about 2 for a $20 \mathrm{~m} / \mathrm{s}$ wind speed.

As an example, Figure 12 gives the cutoff values estimated from a set of imagettes. These values are plotted versus the wind speed inferred from the collocated scatterometer data. The solid line represents fully developed seas while the dash-dotted line is for a fixed fetch condition (developing seas). The agreement is seen to be reasonable. 


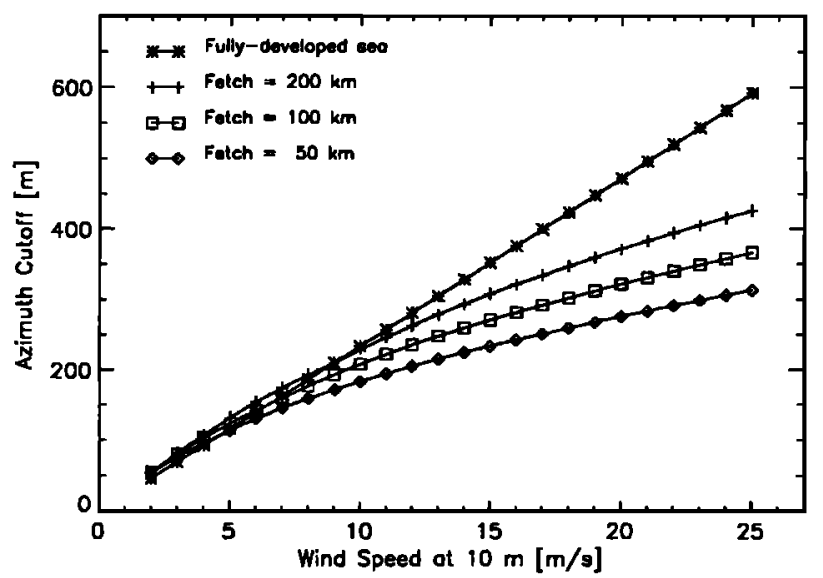

Figure 11. Estimation of the azimuth cutoff value for different wind speeds at $10 \mathrm{~m}$ and different fetch values (unlimited fetch, i.e., fully-developed, 200, 100, and 50 km).

5.2.2. Swell and wave relaxation biases. Apparent to these comparisons between azimuthal cutoff parameters $\lambda$ and scatterometer wind speed, there are cases where the observed $\lambda$ is much higher than the prediction inferred from the scatterometer wind speed. For instance, under light wind conditions, preexisting swell systems will bias the azimuthal cutoff wind speed estimate since the wind algorithm based on the empirical relationship (9) only relies on the definition of a pure wind-wave spectral model (of JONSWAP type). Thus, if we consider a sinusoidal swell system whose wavelength is $l_{0}$ and amplitude is $A$, the additional contribution to the total variance of azimuthal displacement is

$$
\rho_{\xi \xi}(0)=\left(\frac{R}{V}\right)^{2} \frac{A^{2} \omega_{0}^{2}}{4}=\left(\frac{R}{V}\right)^{2} \frac{\pi g A^{2}}{2 l_{0}}
$$

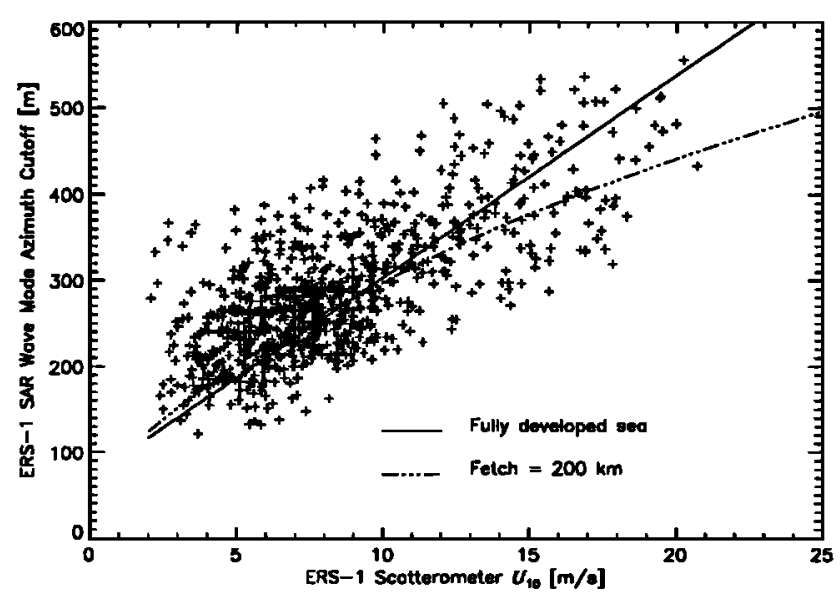

Figure 12. ERS-1 SAR wave mode imagette azimuth cutoff versus $U_{10}$ inferred from collocated ERS-1 scatterometer data (plus). The solid line represents the theoretical relationship between SAR azimuth cutoff and $U_{10}$ for a fully developed sea whereas the dash-dotted line represents a fetch length of $200 \mathrm{~km}$.
Yet, from our comparisons, pure swell contributions to the total smear do not seem to have a strong impact except under light wind conditions $(<6 \mathrm{~m} / \mathrm{s})$. This bias is shown in Figure 13 for a swell of $200 \mathrm{~m}$ wavelength and $1 \mathrm{~m}$ amplitude. This may explain the dispersion of azimuth cutoff values for small wind speeds.

Finally, meteorological fronts can also be sources of overpredicted wind speed with the nominal relationship. Indeed, recall that spaceborne SAR images often exhibit strong gradients and striking contrasts over extended and visually well delineated spatial regions. Such patterns may be associated with a wide variety of oceanic and/or atmospheric phenomena. Under frontal conditions a conjecture is that a scatterometer or a SAR can exhibit rapid and severe attenuations due to the scarcity of capillary-gravity waves, while the azimuthal cutoff measurements which are primarily influenced by still-present longer waves react more smoothly. Such a case can be conceptualized as a low-pass filtering effect, such that some surface motion can be conserved but the Bragg-resonant waves (about $7 \mathrm{~cm}$ for the $\mathrm{C}$ band ERS SARs) almost disappear [Chapron et al., 1994b]. Such a relaxation difference between scales will affect and bias the wind speed estimate from an observed $\lambda$. To resolve this problem, we will investigate the use of other statistical parameters.

5.2.3. Directionality aspects. So far, we have neglected the fact that the azimuth shifts are proportional to the surface velocity component in the direction of the radar beam. In fact, it is the rms line-of-sight orbital velocity that must be considered rather than the rms vertical velocity. One then must write

$$
\lambda=\pi \sqrt{\int_{0}^{\infty} S(k)|T(k)|^{2} d k}
$$

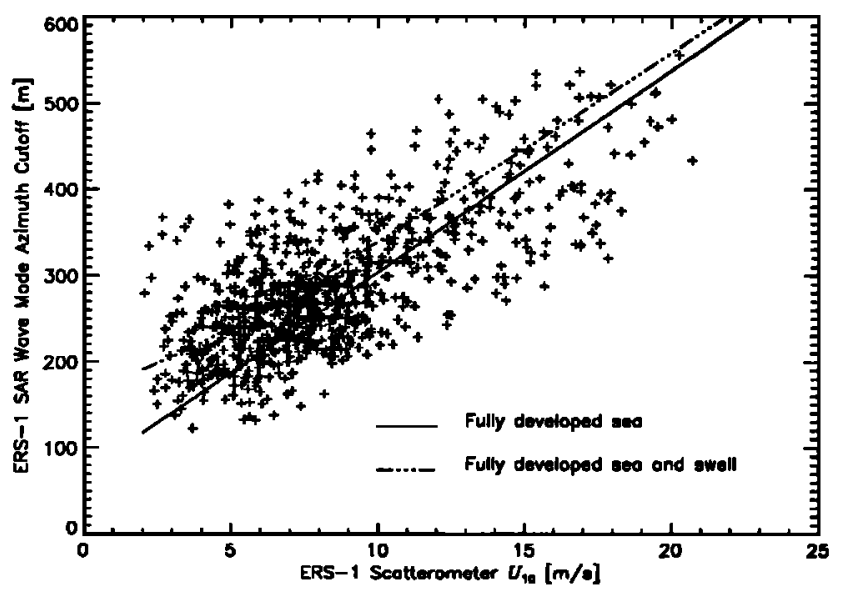

Figure 13. ERS-1 SAR wave mode imagettes azimuth cutoff versus $U_{10}$ inferred from collocated ERS-1 scatterometer data (plus). The solid line represents the theoretical relationship between SAR azimuth cutoff and $U_{10}$ for a fully developed sea whereas the dash-dotted line shows the contribution of an additional sinusoidal swell of wavelength $200 \mathrm{~m}$ and amplitude $1 \mathrm{~m}$. 
where $T(k)$ is the linear transfer function associated with the surface displacement. The transfer function is defined as [Krogstad and Vachon, 1994]

$$
T(k)=\frac{R}{V}|\omega|\left[j \cos \theta+\frac{k_{r}}{k} \sin \theta\right]
$$

where $\theta$ is the local incidence angle and $k_{r}$ is the range wavenumber. If we further suppose that the spreading function of the wave spectral model is represented by a second-order cosine Fourier coefficient, the unidirectional sea spectrum is

$$
S(k, \phi)=\frac{1}{k} S(k) \frac{1}{2 \pi}\left\{1+\Delta(k) \cos \left[2\left(\phi-\phi_{0}\right)\right]\right\}
$$

with $\phi_{0}$ the wind mean direction relative to the range axis. We obtain

$$
\lambda=\pi \frac{R}{V} \sqrt{\int_{0}^{\infty} \omega^{2} S(k) F\left(k, \theta, \phi_{0}\right) d k}
$$

where,

$$
F\left(k, \theta, \phi_{0}\right)=\cos ^{2} \theta+\left(\frac{\sin ^{2} \theta}{2}+\Delta(k) \frac{\sin ^{2} \theta}{4} \cos 2 \phi_{0}\right)
$$

For the ERS SARs the incidence angle is $19.9^{\circ}$ or $23.5^{\circ}$, so $\cos ^{2} \theta+\frac{1}{2} \sin ^{2} \theta>0.92$. Since the absolute value of $\Delta(k)$ is bound by unity, the ratio between range and azimuth cases is at most given by

$$
r=\sqrt{\left(\cos ^{2} \theta+\frac{3}{4} \sin ^{2} \theta\right) /\left(\cos ^{2} \theta+\frac{1}{4} \sin ^{2} \theta\right)}
$$

For $\theta=23.5^{\circ}$ the differences between $\lambda_{\text {range }}$ and $\lambda_{\text {azimuth }}$ are then negligible $(<5 \%)$. The directionality dependence will be weak, and only considering the rms vertical velocity is, at first, a good approximation.

\section{Statistical Analysis}

Besides calibration and azimuth cutoff analysis of ERS-1/2 imagettes, higher-order statistical analysis, up to fourth order, was also carried out. Indeed, the understanding and determination of SAR image distributions remain problems. The results of this study will be discussed in this section as well as the interpretation of the statistical information.

\subsection{ERS-1/2 SAR Imagette Distributions}

The problem of determining the distribution followed by the cross-section or the amplitude of SAR images has been widely studied and several models have been proposed: Rayleigh, K [Jakeman and Pusey, 1976], lognormal [Trunk, 1972], Weibull [Fay et al., 1977], etc. A useful method is to compare their higher-order moments, particularly the couple $\left(\beta_{1}, \beta_{2}\right)$, the skewnesssquared, and the kurtosis.

When the radar illuminates a large area of the sea, it is usually found that the amplitude of the signal is Rayleigh distributed. This is in accordance with the central limit theorem (CLT) since the signal can be thought of as being the sum of a large number of randomly phased, independent scatterers. In the case of a summation process of $N_{l}$ independent looks in amplitude, the distribution will follow the convolution of $N_{l}$ Rayleigh probability density functions (pdf). The couple $\left(\beta_{1}, \beta_{2}\right)$ is given by

$$
\begin{aligned}
& \beta_{1}=\frac{m_{3 c}{ }^{2}}{m_{2 c}{ }^{3}}=\frac{4 \pi(\pi-3)^{2}}{N_{l}(4-\pi)^{3}} \\
& \beta_{2}=\frac{m_{4 c}}{m_{2 c}{ }^{2}}=3+\frac{24 \pi-6 \pi^{2}-16}{N_{l}(4-\pi)^{2}}
\end{aligned}
$$

where $m_{i c}$ represents the centered moment of $i$ th order. For pure speckle ERS imagettes (i.e., with no texture in the scene), $\left(\beta_{1}, \beta_{2}\right)$ is $(0.13,3.08)$. Examples of pure speckle have been found for SAR scenes over lakes or large glacier areas.

Very few imagettes have Rayleigh statistics over the ocean. Indeed, the return signal generally has a more spike-like structure, which leads to an extended tail of the SAR amplitude image pdf. The couple $\left(\beta_{1}, \beta_{2}\right)$ for all ERS-1 and ERS-2 imagettes considered has been plotted in a Pearson diagram (Figure 14) which also shows the different distribution families [Johnson and $K o t z, 1969]$. The data lie on the $K$ law three-looks in amplitude for small values of $\beta_{1}$. For large values of $\beta_{1}$ the data lie between $K$ and lognormal. Moreover, $\beta_{1}$ and $\beta_{2}$ take lower values for ERS-1 imagettes because of ADC saturation effect. This suggests that ADC saturation does not modify the pdf family but rather acts on the range of $\beta_{1}$ and $\beta_{2}$. Note that only a few points are close to the Rayleigh three-looks couple. The evident departure from standard Rayleigh statistics demonstrates the violation of the CLT for most imagettes. Various explanations for such statistics have been proposed, including either non-Bragg scattering contributions [Jakeman and Pusey, 1976] or fluctuations characterized by two spatial coherence lengths due to SAR high-resolution properties [Ward, 1981; Jakeman and Tough, 1988].

\subsection{Contribution of Non-Bragg Scattering}

The extended tail of the SAR pdf has often been interpreted as the result of non-Bragg scattering. Thus, in the case of rough sea states, the cross-section is dominated by sea spike density due to non coherent specular scattering (e.g., wave-breaking events). Tilley and Sarma [1993] suggested that when the cross-section is dominated by sea spike density clipped in amplitude, the cross-section measurement can be approximated by the number of point-scattering processes occurring 


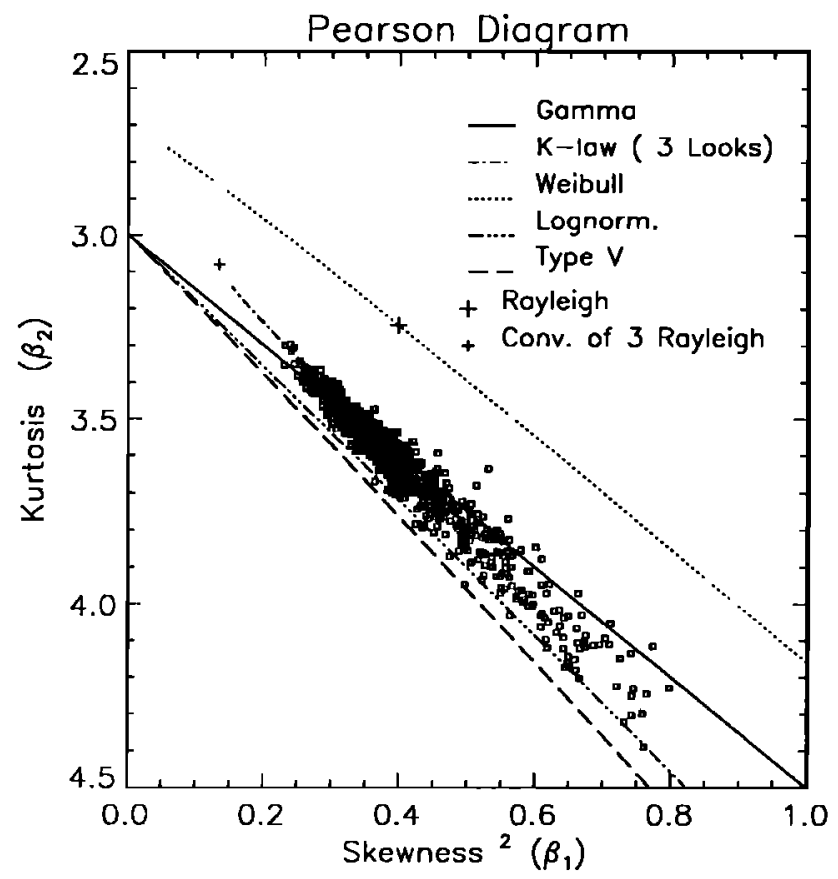

(a)

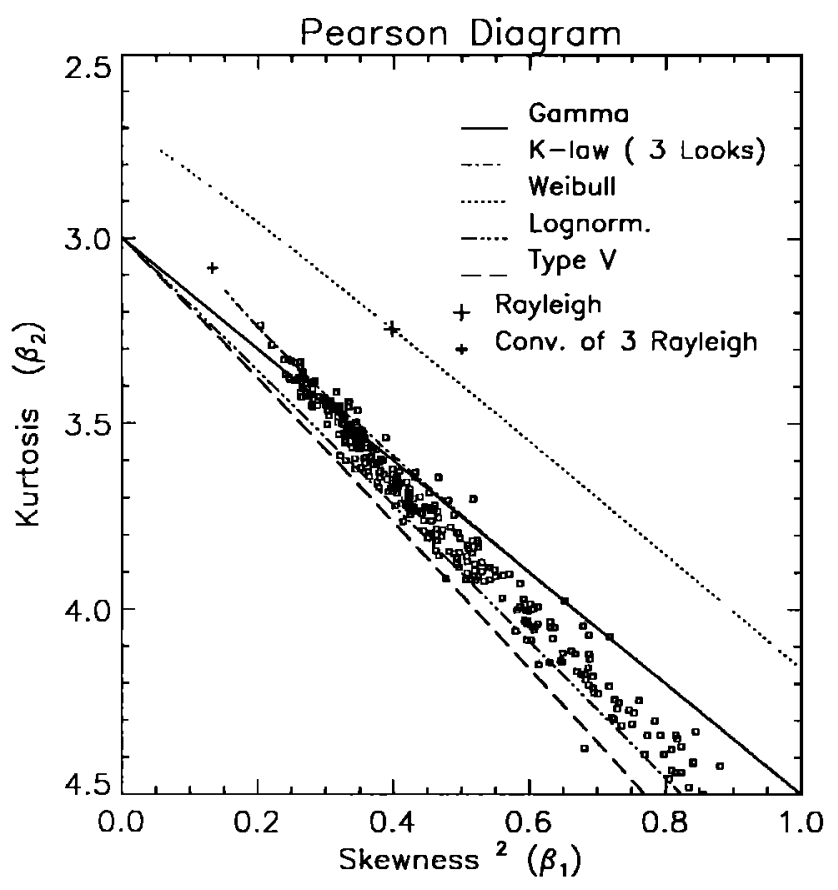

(b)

Figure 14. Imagettes kurtosis versus skewness-squared in a Pearson diagram (squares). (a) ERS-1. (b) ERS-2.

within the integration time. This theory implies that SAR distribution degenerates to a Poisson law. Jakeman and Pusey [1976] suggested that the considerable enhancement of intensity fluctuation can be interpreted in terms of facet-like scattering. Indeed, large fluctua- tions of individual form factors will lead to a decrease in the apparent number of scatterers contributing to the scattering intensity. So far, there has been no attempt to relate SAR statistics to sea spike density. It has been shown that a SAR Doppler spectrum analysis should provide useful information on spike density and time coherence [Kerbaol et al., 1996].

\subsection{Modulation Contribution}

The departure from standard Rayleigh statistics can be interpreted from the SAR high-resolution property. The presence of only a small number of scatterers in the illuminated area might explain the violation of the CLT. However, the existence of pure speckle imagettes, that are well modeled as a Rayleigh three-look distribution, makes this rather unlikely. The average number of scatterers within a resolution cell can then be considered asymptotically large. Nevertheless, long wavelength gravity waves and swell contribute to the distortion of the overall distribution. This concept of large-scale inhomogeneities modulating a dense array of small-scale scatterers has been introduced in various ways in the literature, including the effect of fluctuations of the number of scatterers within a cell [Jakeman and Tough, 1988] or the representation of the two-scale ocean surface by a compound process [Ward, 1981]. Both models lead to the $\mathrm{K}$ law.

Let us consider that the observed SAR power image as being equal to $Y(x)=X(x) S(x)$ where $S$ is the speckle governed by the Rayleigh three-look distribution and $X$ is the unspeckled image containing the ocean wave modulation. If we assume that $S$ and $X$ are independent, the spectrum of $Y$ is given by [Goldfinger, 1982]

$\Phi_{Y}(k)=E[S]^{2} \Phi_{X}(k)+\left[\Phi_{X}(k)+E[X]^{2} \delta(k)\right] \otimes \Phi_{S}(k)$

where $\otimes$ is the convolution operator. Acknowledging that the characteristic correlation distance for the speckle is shorter than for the modulation in $X$, it was suggested that $\Phi_{Y}(k)$ could be approximated as [Krogstad, 1994]

$$
\Phi_{Y}(k)=E[S]^{2} \Phi_{X}(k)+\left[\operatorname{var}(X)+E[X]^{2}\right] \Phi_{S}(k)
$$

Hence, considering the relative wave modulation $m$ and $\chi$ the normalized speckle spectrum such that $\Phi_{S}(k)=$ $\operatorname{var}(S) \chi(k), \Phi_{Y}(k)$ becomes:

$$
\Phi_{Y}(k)=\alpha E[Y]^{2} \chi(k)+E[Y]^{2}\left[\Phi_{m}(k)+\alpha \operatorname{var}(m) \chi(k)\right]
$$

where $\alpha=\sigma_{S}^{2} / E[S]^{2}=.253$ for ERS-1/2 three-look power imagettes ( $\alpha$ is $1 / 3$ for three-look power summation). The first term in (22) represents the underlying speckle spectrum while the second represents the modu- 
lation contribution. SAR power image variance to mean squared ratio is derived from (22) such that

$$
\frac{\operatorname{var}\left(I_{\mathrm{SAR}}\right)}{E\left[I_{\mathrm{SAR}}\right]^{2}}=\alpha+[\operatorname{var}(m)(1+\alpha)]
$$

This last equation illustrates the departure from a Rayleigh three-look distribution, providing a wave system is imaged by the instrument. SAR normalized variances (corrected for ADC saturation) have been plotted as a function of scatterometer wind speed for ERS-1 (Figure 15). Azimuth and range wind direction were selected. One can see that the wind direction can be discriminated when analyzing SAR variance to mean squared ratio. An attempt was made to simulate this parameter using a JONSWAP-like two-dimensional sea spectrum and the modulation transfer functions (MTF) as described by Hasselmann and Hasselmann [1991]. In addition, an average swell system was added to the sea spectrum to account for the fact that modulation was always detected, even at lowest wind speeds. There are still discrepancies between theoretical predictions and observed data. However, most of the salient features are captured by the simulation. Since the RAR modulation transfer function is based on the Bragg-scattering model, discrepancies may be related to the specular contribution. Indeed, at such an incidence angle (about $20^{\circ}$ ), quasi-specular scattering mechanisms cannot be neglected. Thus the analysis of the SAR variance to mean squared ratio parameter should help to improve the RAR MTF definition.

\section{Summary}

The ERS-1 and ERS-2 wave mode imagettes can offer new insights to wind/wave modellers. The interpreta-

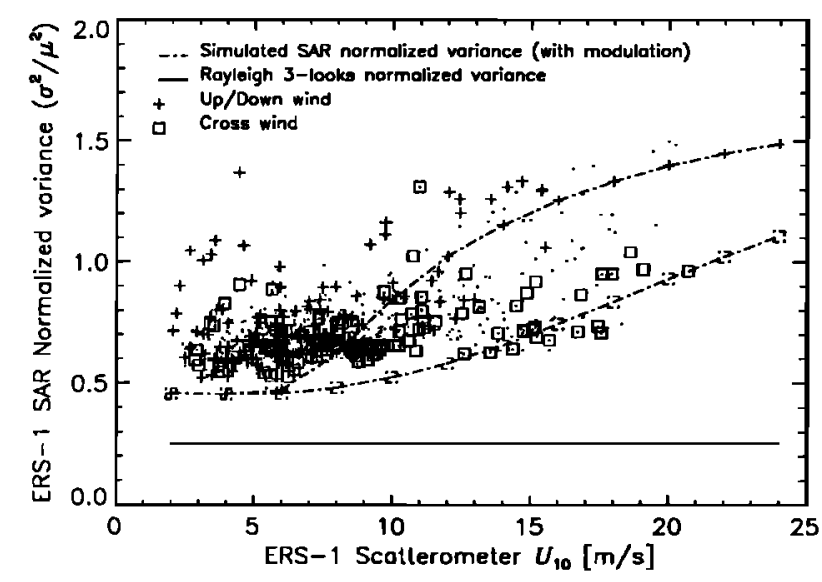

Figure 15. ERS-1 SAR wave mode imagettes power normalized variance versus $U_{10}$ inferred from collocated ERS-1 scatterometer data for wind blowing in range direction (asterisks) and in azimuth direction (squares). The solid line represents the theoretical normalized variance value $(\alpha=0.253)$ corresponding to the square of the convolution of 3 Rayleigh laws and the dash-dotted line represents the simulated SAR normalized variance. tion of ERS-1 SAR wave mode data in terms of normalized radar cross section (NRCS) through intensive comparisons with scatterometer estimates, clearly show saturation effects for ocean scenes. Such a problem seems to be adequately compensated for ERS-2 wave mode products after reduction of onboard gains and for ERS1 after ADC corrections. Our results show that SAR NRCS products can be successfully interpreted using a standard scatterometer wind speed retrieval algorithm. This affirms the use of the SAR instrument as a highresolution scatterometer. The difference in resolution between the SAR and the scatterometer does not appear to present any obstacle to the use of an operational wind speed algorithm.

The along track resolution has been studied using a time-domain method to obtain cutoff wavelength estimates. Observed azimuthal falloff confirmed the predicted limitation of high-altitude synthetic aperture radars. Convincing comparisons highlight the development of a SAR wind algorithm, from a kinematic point of view, that accounts for vertical motions of sea surface (i.e., the directivity can be neglected). This analysis suggests the use of an alternative method to obtain an independent wind speed measurement. However, one needs to be cautious with the estimation bias caused by the inertia of gravity waves in the case of fetch limitation or relaxation effects. Further investigation is required to extend this algorithm to other sensors such as RADARSAT or ENVISAT. In particular, sea surface motions can no longer be reduced to vertical displacements at larger incidence angle, and the directivity cannot be neglected. Moreover, acknowledging that HH polarization SAR instruments are more sensitive to short lifetime, fast-moving scatterers [Jessup et al., 1990; Apel, 1994; Smith et al., 1996], collocation between ERS-2 and RADARSAT SAR data may be used to address this issue.

It was also shown that the statistical distribution of the SAR wave mode products is well characterized by an extended-tail distribution lying between $\mathrm{K}$ law and lognormal law in the Pearson diagram. Furthermore, an attempt was made to reproduce SAR power image variance by simulating the SAR transform of a JONSWAPlike two-dimensional sea spectrum using standard modulation transfer functions as described by Hasselmann and Hasselmann [1991]. This leads to the conclusion that the departure from the standard Rayleigh threelook pdf was mainly due to the SAR high resolution. Further investigations may be envisaged to globally define a standard RAR modulation transfer function to improve SAR inversion algorithms.

To conclude, following the methodology presented here, the analysis of SAR data in the spatial domain rather than exclusively in the spectral domain also offers useful information for operational purposes. An independent wind speed product, based on azimuth cutoff analysis, is proposed. This would complement the scatterometry-type analysis. 
Table A1. Summary of $K^{\prime}$ Values.

\begin{tabular}{lcccc}
\hline Sensor & Number of Imagettes & $K_{\mathrm{dB}}^{\prime}$ & Incidence Angle & Data Set \\
\hline ERS-1 & 1092 & 76.21 & $\theta=19.9^{\circ}$ (prior to June 1995) & North Pacific Ocean \\
ERS-1 & 693 & 77.55 & $\theta=23.5^{\circ}$ (after June 1995) & Indian Ocean \\
ERS-2 & 342 & 73.78 & $\theta=23.5^{\circ}$ (after April 1995) & Indian Ocean \\
\hline
\end{tabular}

\section{Appendix: Calibration Constant Retrieval}

Following the assumption of a 1-to-1 ratio between $I_{\mathrm{SAR}}-$ Loss $_{\mathrm{dB}}-K_{\mathrm{dB}}^{\prime}$ and $\sigma^{\circ} \mathrm{SCAT}$, the mean square estimator for $K^{\prime}$ is given by

$$
K^{\prime}=\frac{1}{n} \sum_{i=1}^{n}\left(I_{\mathrm{SAR}}-\operatorname{Loss}_{\mathrm{dB}}-\sigma^{\circ} \mathrm{SCAT}\right)
$$

This estimator is expected to be consistent for both data sets over the Indian Ocean (ERS-1 and ERS-2 at $23.5^{\circ}$ ). In the case of the North Pacific Ocean (ERS-1 at $\left.19.9^{\circ}\right)$ the estimator should only be applied to $\sigma^{\circ}$ values of scatterometer that are below $-2 \mathrm{~dB}$ (above this level, ADC correction is not expected to give satisfactory results). Table A1 presents the results of this estimator for both incidence angle configurations of ERS-1 and for ERS-2.

Acknowledgment. This work was partly supported by a contract from the European Union, program MAST, entitled European Community Atmosphere Wave Ocean Model (ECAWOM), MAS2-CT94-0091.

\section{References}

Alpers, W.R., and C. Brüning, On the relative importance of motion-related contributions to the SAR imaging mechanism of ocean surface waves, IEEE Trans. Geosci. and Remote Sens., GE-24(6), 873-885,1986.

Alpers, W.R., and C.L. Ruffenach, The effect of orbital motions on synthetic aperture radar imagery of ocean waves, IEEE Trans. Antennas Propag., AP-27(5), 685-690, 1979.

Alpers, W.B., D.B. Ross, and C.L. Ruflenach, On the detectability of ocean surface waves by real and synthetic aperture radar, J. Geophys. Res., 86(C7), 6481-6498, 1981.

Apel, J.R., An improved model of the ocean surface wave vector spectrum and its effect on radar backscatter, $J$. Geophys. Res., 99(C8), 16269-16291, 1994.

Bao, M., C. Brüning, and W. Alpers, A generalized nonlinear ocean wave SAR spectral integral transform and its application to ERS-1 SAR ocean wave imaging., in Proceedings Second ERS-1 Symposium, 219-224, ESA Publications Division, ESTEC, Noordwijk, The Netherlands, 1994.

Beal, R.C., D.G. Tilley, and F.M. Monaldo, Large and smallscale spatial evolution of digitally processed ocean wave spectra from Seasat synthetic aperture radar, J. Geophys. Res., 88(C3), 1761-1778, 1983.

Brillinger, D.R., Distributions of particle displacements via higher-order moment functions, Proc. IEEE, Part. F, 140(6), 390-394, 1993.

Brüning, C., W. Alpers, and K. Hasselmann, Monte-Carlo simulations studies of the non-linear imaging of a twodimensional surface wave field by a synthetic aperture radar, Int. J. Remote Sens., 11(10), 1695-1727, 1990.

Brüning, C., S. Hasselmann, and K. Hasselmann, First evaluation of ERS-1 synthetic aperture radar wave mode data, Global Atmos. Ocean Syst., 2, 61-98, 1994.

Chapron, B., R. Garello, V. Kerbaol, and J.M. Lefevre, Nonlinear theory of ocean-SAR transformation and statistical analysis of ERS-1 SAR wave mode imagettes, in Proceedings Second ERS-1 Symposium, 247-250, ESA Publications Division, ESTEC, Noordwijk, The Netherlands, 1994a.

Chapron B., D. Vandemark, and F.C. Jackson, Airborne measurements of the ocean's Ku-band radar cross-section at low incidence angle, Atmos. Ocean, 32(1), 179-193, 1994b.

Chapron, B., T. ElFouhaily, and V. Kerbaol, Calibration and validation of ERS wave mode products, Tech. Rep. DRO/OS/95-02, Inst. Fr. de Rech. pour l'Exploit. de la Mer, Brest, France, 1995.

ElFouhaily, T., B. Chapron, K. Katsaros, and D. Vandemark, A unified directional spectrum for long and short wind-driven waves, J. Geophys. Res., 102(C7), 15781$15796,1997$.

Engen, G., and H. Johnsen, Analysis and inversion of ERS1 image cross-spectra, in IGARSS'95, 1863-1865, IEEE Press, Piscataway, N.J., 1995.

European Space Agency, ERS-1 system, Eur. Space Agency Spec. Publ., ESA SP-1146, 26-27, 1992a.

European Space Agency, ESA ERS-1 product specification, Eur. Space Agency Spec. Publ., ESA SP-1149, 1992b.

Fay, F.S., J. Clarke, and R.S. Peters, Weibull distribution applied to sea clutter, IEE Conf. Publ., 105, 101-104, 1977.

Goldfinger, A.D., Estimation of spectra from speckled images, IEEE Trans. Aerosp. Electron. Syst., AES-18(5), 675-681, 1982.

Hasselmann, K., and S. Hasselmann, On the nonlinear mapping of an ocean wave spectrum into a synthetic aperture radar image spectrum and its inversion, J. Geophys. Res., 96(C6), 10713-10729, 1991.

Hasselmann, K., R.K. Raney R.K., W.J. Plant, W. Alpers, R.A. Shuchman, D.R. Lyzenga, C.L. Rufenach, and M.J. Tucker, Theory of synthetic aperture radar ocean imaging: A MARSEN view, J. Geophys. Res., 90(C3), 4659-4686, 1985.

Jackson, F.C. and C.Y. Peng, Comment on "Imaging radar observations of directional properties of ocean waves" by W. McLeish and D.B. Ross, J. Geophys. Res., 90(C4), 7367-7370, 1985.

Jakeman, E., and P.N. Pusey, A model for non-Rayleigh sea echo, IEEE Trans. Antennas Propag., AP-24(6), 806-814, 1976.

Jakeman, E., and R.J.A. Tough, Non-gaussian models for the statistics of scattered waves, Adv. Phys., $37(5), 471-$ $529,1988$.

Jessup, A.T., W.C. Keller, and W.K. Melville, Measurements of sea spikes in microwave backscatter at moderate incidence, J. Geophys. Res., 95(C6), 9679-9688, 1990. 
Johnson, N.L., and S. Kotz, Distribution in Statistics: Continuous Univariate Distributions, vol. I, John Wiley, New York, 1969.

Kanevsky, M.B., On the theory of SAR ocean wave imaging, IEEE Trans. Geosci. and Remote Sens., 31(5), 1031-1035, 1993.

Kerbaol, V., and B. Chapron, Calibration and validation of ERS-1/2 wave mode products, Eur. Space Agency Rep. PO NO 160709, Inst. Fr. de Rech. pour l'Exploit. de la Mer, Brest, France, October 1996.

Kerbaol, V., B. Chapron, T. ElFouhaily, and R. Garello, Fetch and wind dependence of SAR azimuth cutoff and higher order statistics in a mistral wind case, in IGARSS'96, 621-624, IEEE Press, Piscataway, N.J., 1996.

Korsbakken, E., Quantitative wind field retrievals from ERS SAR images, Tech. Rep., Eur. Space Agency (ESTEC), 1996.

Krogstad, H.E., A simple derivation of hasselmann's nonlinear ocean-synthetic aperture radar transform, J. Geophys. Res., 97(C2), 2421-2425, 1992.

Krogstad, H.E., Some comments about the processing of ERS-1/2 imagettes and the wave mode product, Tech. Rep. STF10 A94008, SINTEF Industrial Mathematics, Trondheim, Norway, 1994.

Krogstad, H.E., and P.W.Vachon, Generalizations of the non-linear ocean-SAR transform and a simplified SAR inversion algorithm, Atmos. Ocean, 32(1), 61-82, 1994.

Laur, H., P.J. Meadows, J.I. Sanchez, and E. Dwyer, ERS-1 SAR radiometric calibration, in Proceedings of the CEOS $S A R$ Calibration Workshop, 257-281, ESTEC, Noordwijk, The Netherlands, ESA WPP-048, 1993.

Laur, H., P. Bally, P. Meadows, J. Sanchez J., B. Schaettler, and E. Lopinto, ERS SAR calibration - Derivation of backscattering coefficient in ESA ERS SAR PRI products, Tech. Note ES-TN-RS-PM-HL09, Eur. Space Agency Eur. Space Res. Inst., Frascaty, Italy, 1996.

Lyzenga, D.R., Numerical simulation of synthetic aperture radar image spectra for ocean waves, IEEE Trans. Geosci. and Remote Sens., GE-24(6), 863-872, 1986.

Ouchi, K., and R.A. Cordey, Statistical analysis of azimuth streaks observed in digitally processed CASSIE imagery of sea surface, IEEE Trans. Geosci. and Remote Sens., 29(5), 727-735, 1991.

Quilfen, Y., ERS-1 off-line wind scatterometer products, Tech. Rep. IFREMER-SCAT/IOA/DOS-01, Inst. Fr. de Rech. pour l'Exploit. de la Mer, Brest, France, 1993.

Scoon, A., I.S. Robinson, and P.J. Meadows, Demonstration of an improved calibration scheme for ERS-1 SAR imagery using a scatterometer wind model, Int. J. Remote Sens., $17(2)$, 413-418, 1996.
Smith, M.J., E.M. Poulter, and J.A. McGregor, Doppler radar measurements of wave group and breaking waves, J. Geophys. Res., 101(C6), 14269-14282, 1996.

Steinberg, H., P.M. Schulteiss, C.A. Wogrin, and F. Zweig, Short-time frequency measurements of narrow-band random signals by means of a zero-counting process, J. Appl. Phys., 26(2), 195-201, 1955.

Stoeffelen, A., and D.L.T. Anderson, ERS-1 scatterometer data characteristics and wind retrieval skill, Adv. Space Res., 13, 553-560, 1993.

Swift, C.T., and L.R. Wilson, Synthetic aperture radar imaging of moving ocean waves, IEEE Trans. Antennas Propag., AP-27(6), 725-729, 1979.

Tilley, D.G., and Y.V. Sarma, A comparison of synthetic aperture radars applied for satellite remote sensing of the ocean surface, Trends Geophys. Res., 2, 467-486, 1993.

Tournadre, J., and S. Blanquet S., Wind and wave mesoscale variability from in-situ and altimeter data, Global Atmos. Ocean Syst., 2, 221-247, 1994.

Trunk, G.V., Radar properties of non-Rayleigh sea clutter, IEEE Trans. Aerosp. Electron. Syst., AES-8(2), 196-204, 1972.

Tucker, M.J., The imaging of waves by satelliteborne synthetic aperture radar: the effect of sea-surface motion, Int. J. Remote Sens., 6(7), 1059-1074, 1985.

Vachon, P.W., and F.W. Dobson, Validation of wind vector retrieval from ERS-1 SAR images over the ocean, Global Atmos. Ocean Syst., 5, 177-187, 1996.

Vachon, P.W., H.E. Krogstad, and J.Scott Paterson, Airborne and spaceborne synthetic aperture radar observations of ocean waves, Atmos. Ocean, 32(1), 83-112, 1994.

Vesecky, J.F., and R.H. Stewart, The observation of ocean surface phenomena using imagery from the Seasat synthetic aperture radar, J. Geophys. Res., 87(C5), 33973430, 1982.

Ward, K.D., Compound representation of high resolution sea clutter, Electron. Lett., 17, 561-563, 1981.

B. Chapron and V. Kerbaol, Département d'Océanographie Spatiale, IFREMER, centre de Brest, BP 70, 29280 Plouzané, France. (e-mail: Bertrand.Chapron@ifremer.fr; Vincent.Kerbaol@ifremer.fr)

P. W. Vachon, Canada Centre for Remote Sensing, 588 Booth Street, Ottawa, Ont. K1A OY7, Canada. (e-mail: vachon@ccrs.emr.ca)

(Received December 9, 1996; revised May 16, 1997; accepted May 29, 1997.) 